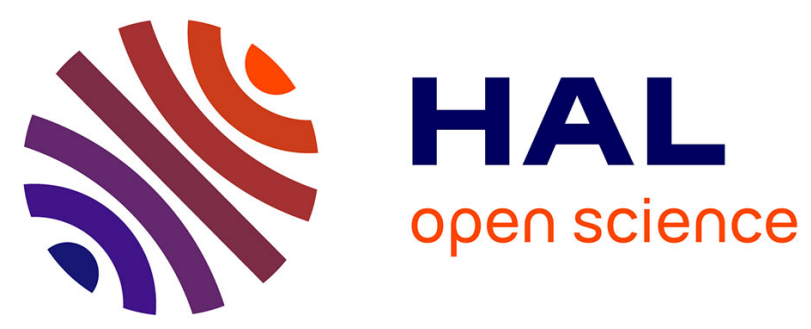

\title{
Dynamic and capillary shear rheology of natural fiber-reinforced composites
}

\author{
Nicolas Le Moigne, Martien van den Oever, Tatiana Budtova
}

\section{To cite this version:}

Nicolas Le Moigne, Martien van den Oever, Tatiana Budtova. Dynamic and capillary shear rheology of natural fiber-reinforced composites. Polymer Engineering and Science, 2013, 53 (12), pp.2582-2593. 10.1002/pen.23521 . hal-00800842

\section{HAL Id: hal-00800842}

https://hal-mines-paristech.archives-ouvertes.fr/hal-00800842

Submitted on 25 Jan 2021

HAL is a multi-disciplinary open access archive for the deposit and dissemination of scientific research documents, whether they are published or not. The documents may come from teaching and research institutions in France or abroad, or from public or private research centers.
L'archive ouverte pluridisciplinaire HAL, est destinée au dépôt et à la diffusion de documents scientifiques de niveau recherche, publiés ou non, émanant des établissements d'enseignement et de recherche français ou étrangers, des laboratoires publics ou privés. 


\title{
Dynamic and Capillary Shear Rheology of Natural Fiber-Reinforced Composites
}

\author{
Nicolas Le Moigne, ${ }^{1,2}$ Martien van den Oever, ${ }^{2}$ Tatiana Budtova ${ }^{1}$ \\ ${ }^{1}$ Mines ParisTech, CEMEF - Centre de Mise en Forme des Matériaux, CNRS UMR 7635, BP 207, \\ 1 rue Claude Daunesse, 06904 Sophia Antipolis Cedex, France \\ ${ }^{2}$ Wageningen UR - Food and Biobased Research, PO Box 17, 6700 AA Wageningen, The Netherlands
}

An extended dynamic and capillary rheological study of molten flax and sisal polypropylene (PP) composites was performed. Fiber concentration varied from 20 to $50 \mathrm{wt} \%$ and shear rate from $0.1 \mathrm{rad} \mathrm{s}^{-1}$ to $10,000 \mathrm{~s}^{-1}$. Maleic anhydride-grafted-PP was used as compatibilizer; it strongly reduces PP and composite viscosity. Composites are yield-stress shear-thinning fluids with solid-like behavior being more pronounced at high fiber content. Composites do not obey Cox-Merz rule, which was explained by different macrostructures of the molten composites in parallel plates and capillary die geometries: random fiber orientation versus strong alignment in the flow direction, respectively. Theories describing the viscosity of suspensions of solid particles were applied to the composites studied and rheological parameters and maximal packing fiber volume fraction were calculated.

\section{INTRODUCTION}

The use of natural fibers as a reinforcing component of polymer matrices is an alternative to classical glass fibers, the former presenting several advantages. Natural fibers are derived from natural and renewable resources, they require less fossil energy during production, they are of lower density, and their processing is much less abrasive. This class of composites has recently been boosted mainly due to the needs of transport and building industries.

Correspondence to: Tatiana Budtova; e-mail: Tatiana.Budtova@minesparistech.fr

Nicolas Le Moigne is currently at Centre des Matériaux des Mines d'Alès (C2MA), Ecole des Mines d'Alés, 6 avenue de Clavières, F30319 Alès CEDEX, France.

CEMEF and Wageningen UR are members of the European Polysaccharide Network of Excellence (EPNOE), www.epnoe.eu.

Contract grant sponsor (for NLM): Carnot M.I.N.E.S. Institute and European Polysaccharide Network of Excellence (EPNOE).
In the last few decades, a lot of attention was paid on correlating composite formulation (type of fibers, their properties, use or not of compatibilizer, etc.) and their final mechanical properties [1-3]. The studies of composite rheology are scarce despite the importance of understanding and predicting the flow and viscoelastic properties of molten composites. The exception is wood polymer composites (WPCs) that are produced on the industrial scale; their rheological and mechanical properties are extensively investigated [4-8]. However, fiber aspect ratio in WPC is very low when compared with other natural fibers, which limits the overall understanding of the viscoelastic properties of natural fiber-reinforced composites. Few studies have been focused on the rheology of composites reinforced with technical natural fibers as flax, hemp, or jute [9-13]. It was shown that these composites demonstrate rheological behavior different from glass fiber-reinforced materials. For example, Basu et al. [9] reported that jute-reinforced isotactic polypropylene (PP) composites present lower shear viscosity than the same PP reinforced with glass fibers because of the better adhesion between glass fiber and PP. However, the authors did not analyze the jute and glass fiber size distribution in the composites, which should also explain their different rheological behaviors. More recently, several studies have shown that the rupture mechanisms of natural fibers on compounding are different from glass fibers [14]. For example, flax breakage is initiated at defects localized along the fibers (so called "kink bands"). In overall, rupture is a result of accumulated fatigue damage due to repeated compressions, bending and tractions during the compounding process [14], leading to wide fiber size and shape distributions with numerous little particles [15]. The necessity to use a compatibilizer to compensate the lack of affinity between the natural fibers and the matrix was also considered as having an important effect on composite rheological behavior, producing a plasticizing effect [10].

It is known from classical suspensions and glass fiber molten composites that the rheological response strongly depends on particle/fiber concentration, orientation, shape, 
and aspect ratio. Natural fiber-reinforced composites are "difficult" materials for performing a rheological analysis: natural fibers can exist in the form of elementary plant cell fibers as well as in bundles, and each type of fiber differs from another in terms of shape, polydispersity, flexibility, and capability to form agglomerates. Composite viscoelastic response also depends on the measurement method: elongational, capillary, or dynamic rheometry should induce different structures in the molten composites as outlined by Mobuchon et al. [16] for short glass fiber-reinforced PP composites.

The aim of this article is to perform an extended rheological characterization of flax-reinforced PP composites of various concentrations; sisal-based composites are also shown for comparison. Both dynamic and capillary shear rheometry are used to cover a wide range of shear rates, from $0.1 \mathrm{rad} \mathrm{s}^{-1}$ to $10,000 \mathrm{~s}^{-1}$. This allows approaching different polymer processing conditions from compression molding to extrusion or injection. As far as some literature mentions the influence of compatibilizer on composite viscosity, we start our study with investigation of the viscoelastic properties of the composite matrix, PP mixed with maleic anhydride-grafted polypropylene (MAPP). We show the large effect of MAPP on blend viscosity and we use this result in the subsequent analysis of composite rheology. Then, we present oscillatory and capillary results and demonstrate the difference of composite structure and rheological properties obtained in plate-plate and capillary die geometry. Finally, we analyze separately oscillatory and capillary results and apply theories developed for suspensions of rigid particles with various aspect ratios and volume fractions to the experimental data obtained.

\section{MATERIALS AND METHODS}

\section{Materials}

Polymer matrix was PP homopolymer PP 575P from $\mathrm{SABIC}^{\circledR}$ for injection molding (pellets, melt flow rate = $10.5 \mathrm{~g} / 10 \mathrm{~min}$ at $230^{\circ} \mathrm{C}$ and $2.16 \mathrm{~kg}$ ISO 1133). The fiber/matrix compatibilizer was MAPP Epolene ${ }^{\circledR}$ G-3015 (now called "Eastman ${ }^{\circledR} G-3015$ ") supplied by Eastman Chemical Company (pellets, $M_{\mathrm{n}}=24,800 \mathrm{~g} / \mathrm{mol}, M_{\mathrm{w}}=$
47,000 g/mol, $3.1 \mathrm{wt} \% \mathrm{MA})$. The proportion between fiber and compatibilizer was $1 \mathrm{wt} \%$ of MAPP per $10 \mathrm{wt} \%$ fibers, as suggested by Snijder and Bos [17] to be the most efficient compatibilization between the natural fibers and the matrix.

Two types of fibers were used: most of the work was done with flax bast fibers from Holstein Flachs (Mielsdorf, Germany). Sisal leaf fibers were used in some cases for comparison with flax-based composites. Sisal was from a sisal rope supplied by Carl Kohl $\mathrm{GmbH} \& \mathrm{Co}$. KG (Hamburg, Germany). Flax fiber was used as received. Sisal fibers were cut using a Retsch SM1 cutting mill with a $10 \mathrm{~mm}$ screen. The average fiber length was $15 \pm 5 \mathrm{~mm}$ before compounding. Fibers were stored in a conditioning room at $19^{\circ} \mathrm{C}$ and $30 \%$ relative humidity. In these conditions, the moisture content was 10.15 and $10.6 \%$ for flax and sisal, respectively. Before compounding, fibers were dried for $1 \mathrm{~h}$ at $105^{\circ} \mathrm{C}$. Composites with fiber contents of 20,30,40, and $50 \mathrm{wt} \%$ of dry matter were prepared. Table 1 presents composite characteristics including median fiber length $L$, diameter $D$, and aspect ratio $L / D$, weighted in number (indicated with subscript "n") and in surface (with subscript "s"), taken from Ref. [15], where fiber size distributions in the same composites were reported.

\section{Composites Preparation}

The composites were prepared as described in our previous study [15]. Briefly, a Haake Rheomix 3000p batch kneader equipped with roller-rotors 3000 rotating at 100 rpm at a set temperature of $185^{\circ} \mathrm{C}$ was used. First, PP and MAPP were introduced in the chamber and mixed for $2.5 \mathrm{~min}$ when a constant torque was reached. Then fibers were added in small amounts during the next 8.5-9 min under continuous blending. After the final portion of fibers was added into the chamber, the blending was continued for 2 min more. Ready composites were collected from the chamber, cooled in the air at room temperature, and converted into pellets using a granulator from KT Handling.

Pellets were dried $24 \mathrm{~h}$ at $105^{\circ} \mathrm{C}$ under vacuum before the rheological experiments. For dynamic shear rheology, dried pellets were converted into discs of $25 \mathrm{~mm}$ in diameter and $2 \mathrm{~mm}$ in thickness using compression molding. The

TABLE 1. Sample designation, composition, and median fiber length, diameter, and aspect ratio weighted in number $\left(L_{\mathrm{n}}, D_{\mathrm{n}},(L / D)_{\mathrm{n}}\right)$ and in surface $\left(L_{\mathrm{s}}, D_{\mathrm{s}},(L / D)_{\mathrm{s}}\right)[15]$.

\begin{tabular}{|c|c|c|c|c|c|c|c|c|c|c|c|}
\hline \multirow[b]{2}{*}{ Sample } & \multirow[b]{2}{*}{$\mathrm{PP}(\mathrm{wt} \%)$} & \multicolumn{2}{|c|}{ MAPP (wt $\%$ ) } & \multicolumn{2}{|c|}{ Fiber content } & \multicolumn{2}{|c|}{$L(\mu \mathrm{m})$} & \multicolumn{2}{|c|}{$D(\mu \mathrm{m})$} & \multicolumn{2}{|c|}{$L / D$} \\
\hline & & In composite & Calculated toward PP & $\mathrm{wt} \%$ & Volume fraction & $L_{\mathrm{n}}$ & $L_{\mathrm{s}}$ & $D_{\mathrm{n}}$ & $D_{\mathrm{s}}$ & $(L / D)_{\mathrm{n}}$ & $(L / D)_{\mathrm{s}}$ \\
\hline $20 \%$ flax/PP & 78 & 2 & 2.5 & 20 & 0.14 & 65 & 196 & 11.6 & 19 & 5.2 & 9.0 \\
\hline $30 \%$ flax/PP & 67 & 3 & 4.3 & 30 & 0.22 & - & - & - & - & - & - \\
\hline $40 \%$ flax/PP & 56 & 4 & 6.7 & 40 & 0.303 & 76 & 167 & 12.3 & 19 & 5.5 & 8.3 \\
\hline $50 \%$ flax/PP & 46 & 5 & 10 & 50 & 0.395 & - & - & - & - & - & - \\
\hline $20 \%$ sisal/PP & 78 & 2 & 2.5 & 20 & 0.140 & - & - & - & - & - & - \\
\hline $40 \%$ sisal $/ \mathrm{PP}$ & 56 & 4 & 6.7 & 40 & 0.303 & 46 & 650 & 16.8 & 123 & 2.8 & 5.4 \\
\hline
\end{tabular}


following procedure was used: $10 \mathrm{~min}$ at $200^{\circ} \mathrm{C}$ under low pressure, followed by applying 10 ton of pressure for $5 \mathrm{~min}$ and subsequent cooling under 20 ton. The discs and pellets were stored under vacuum to protect them from humidity.

\section{Dynamic Rheology}

The rheological properties of PP/MAPP binary blends and natural fibers/PP/MAPP composites in dynamic oscillation mode were carried out using a strain controlled rheometer ARES (TA Instrument) equipped with a parallel plate geometry. The upper and lower plates were 25 and $50 \mathrm{~mm}$ in diameter, respectively. All experiments were carried out at $200^{\circ} \mathrm{C}$ under predried nitrogen flow to reduce thermal degradation, if any. When placed between the two hot plates with in-between distance of $2.3 \mathrm{~mm}$, the discs were slightly swelling in the molten state and a normal force was recorded. The gap was progressively set to $2 \mathrm{~mm}$ and a delay of 2000 $\mathrm{s}$ was applied to the molten composites to reach the equilibrium (zero normal force) before starting frequency sweeps.

As discussed by Mobuchon et al. [16], the gap between the two plates must be several times greater than the fiber length to avoid wall effects. For example, Mobuchon et al. [16] used gap-to-fiber length ratio of 3, whereas Bibbo et al. [18] used a minimum ratio of 1 . According to our study performed on the statistical analysis of fiber size and aspect ratio distribution in the same composites [15], the median fiber lengths weighted in surface are 167 and $650 \mu \mathrm{m}$ for 40 wt $\%$ flax and $40 \mathrm{wt} \%$ sisal based composites, respectively (see Table 1). Surface-weighted values are considered here as they take into account long fibers contrary to numberweighted values, as far as long fibers determine the required gap. No fibers exceeding $600 \mu \mathrm{m}$ were obtained for flax based composites [15]. Sisal fibers contain a certain fraction of bundles exceeding $2000 \mu \mathrm{m}$ that is negligible in number, $0.06 \%$, and represent $6.2 \%$ in surface [15]. The reason of the presence of nondissociated and long sisal bundles is the high content of lignin, contrary to flax. As far as most of our study is performed on flax-based composites, a gap of 2 $\mathrm{mm}$ was considered to be sufficient to neglect wall effects.

Frequency sweep tests were performed from 100 to 0.1 $\operatorname{rad} s^{-1}$. The linear viscoelastic domain was checked for all composites and the minimum limit strain recorded for the highest concentration was $0.1 \%$. This strain value was used for all experiments. The measurements were done twice for each blend and composite and were well reproducible. The thermal stability at $200^{\circ} \mathrm{C}$ under nitrogen was higher than $2 \mathrm{~h}$.

\section{Capillary Rheology}

Measurements of the shear viscosity of PP/MAPP binary blends and natural fibers/PP/MAPP composites were carried out at $200^{\circ} \mathrm{C}$ with a Rosand capillary rheometer RH7-2 (Malvern) with a $12 \mathrm{~mm}$ barrel. The capillary die used has an aspect ratio of 16 (length $16 \mathrm{~mm}$ to diameter of $1 \mathrm{~mm}$ ) with $90^{\circ}$ entrance angle. The compounded pel- lets were filled in the barrel in four times applying a pressure of $1 \mathrm{MPa}$ between each filling. The total filling time was around $9 \mathrm{~min}$. Before measurements, the material was heated in the barrel for 6 min while applying a pressure of $1 \mathrm{MPa}$ at time $t=0$ and $t=3 \mathrm{~min}$. The pressure was measured for piston speeds varying from 400 to 2 $\mathrm{mm} / \mathrm{min}$ corresponding to apparent shear rates $\dot{\gamma}_{a}$ varying from 7680 to $38 \mathrm{~s}^{-1}$.

The Bagley correction was applied to account for the pressure drop occurring at the entrance and exit regions of the capillary. A "zero length" capillary die was used to correct the pressure data and determine the true shear stress $\tau$ occurring inside the capillary. The Rabinowitsch analysis was also applied to the data to calculate the true shear rate at the wall:

$$
\dot{\gamma}=\dot{\gamma}_{a}\left(\frac{3 k+1}{4 k}\right)
$$

where $k$ is the flow index corresponding to the slope of $\tau=f\left(\dot{\gamma}_{a}\right)$ correlation. The shear viscosity $\eta$ is defined as the ratio of true shear stress to true shear rate, $\tau / \dot{\gamma}$.

\section{Scanning Electron Microscopy Observations}

The cross-sections of discs made for rotational rheometry experiments and of extrudates from capillary rheometry experiments were evaluated with a scanning electron microscope Philips XL30 ESEM LaB6. The observation conditions were the following: pressure 0.2-0.3 mbar, acceleration voltage $15 \mathrm{keV}$.

\section{RESULTS AND DISCUSSION}

\section{Influence of MAPP on the Rheological Properties of PP/MAPP Binary Blends}

Incorporating MAPP in composites is a classical method of improving the adhesion between matrix and fibers. It has proved its efficiency assuming that the quantity of MAPP is well adjusted to fiber concentration [10, 17, 19]. The addition of MAPP leads to two phenomena: on one hand, it reinforces fiber-matrix interactions [13], and on the other hand, it decreases matrix viscosity if present as free unbounded molecules. To understand and predict rheological properties of such composite, the following questions should be answered: (i) Does MAPP significantly influence matrix (MAPP/PP) viscosity? (ii) How to predict MAPP/PP viscosity?

As it was reported by Twite-Kabamba et al. [10], for a hemp-MAPP-PP composite, at low fiber concentrations (i.e., $<20 \mathrm{wt} \%$ ), the presence of $2 \%$ MAPP counterbalances the viscosity increase due to incorporation of hemp fibers. Composite viscosity becomes then comparable to that of pure PP. The fact that at low fiber concentrations composite viscosity can be the same or even lower than 
that of pure PP without compatibilizer means that there is a lot of free MAPP chains not grafted on fiber surface. Besides, it was shown that in some conditions MAPP can form droplets of a separate phase when mixed with PP [20]. Natural fiber-reinforced PP composites compatibilized with MAPP can be thus considered as a ternary system composed of fibers with bound and unbound MAPP randomly dispersed in a PP matrix.

Complex viscosity $\eta^{*}$ from oscillation measurements as a function of frequency $\omega$ and shear viscosity $\eta$ from capillary measurements as a function of shear rate $\dot{\gamma}$ for pure PP, pure MAPP and their mixtures of various compositions are presented in Fig. 1. Both polymers and their mixtures show a classical pseudoplastic behavior and the Cox-Merz rule is obeyed, i.e., complex and shear viscosity coincide. The same was reported for neat PP; see for example Ref. [16]. It is also clear that the addition of MAPP significantly decreases PP viscosity, as expected considering the lower viscosity of MAPP. Such decrease in PP/MAPP mixture viscosity has also been reported by [21, 22]; however, in certain conditions they found that Cox-Merz rule was not obeyed which was attributed to a phase separation between PP and MAPP.

To evaluate the influence of MAPP concentration on blend viscosity, zero-shear rate viscosity was determined for each blend using Carreau-Yasuda model:

$$
\eta(\omega, \dot{\gamma})=\eta_{0}\left[1+(\lambda(\omega, \dot{\gamma}))^{a}\right]^{(n-1) / a}
$$

where $\eta_{0}$ is the zero-shear rate viscosity, $\lambda$ is a characteristic time, $a$ is the Yasuda parameter, and $n$ is the flow index describing the shear-thinning region. First, the flow

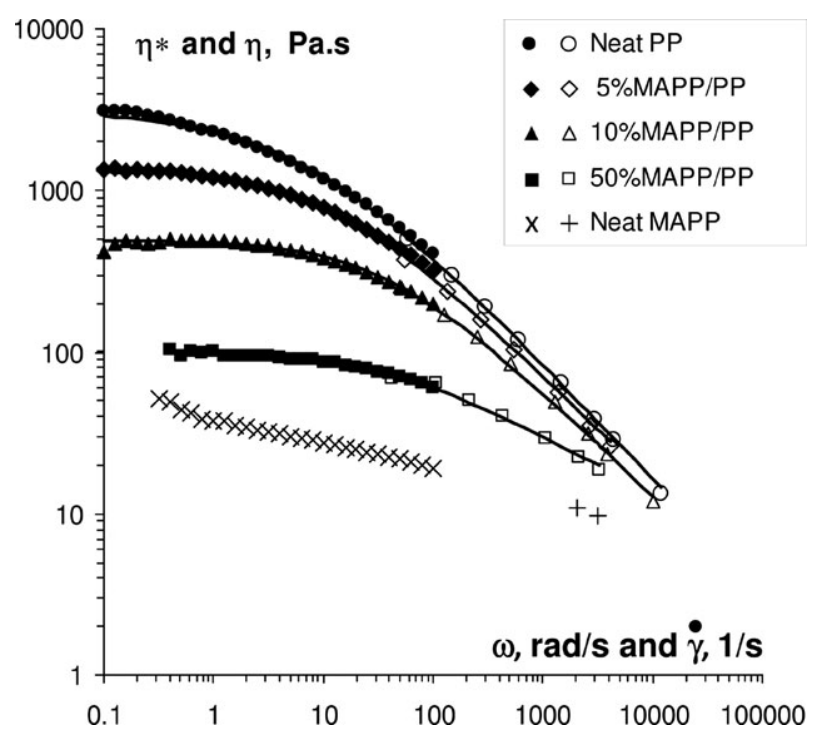

FIG. 1. Complex and shear viscosity of PP, MAPP, and their blends obtained from oscillation (filled symbols) and capillary (open symbols) measurements. Solid lines are the corresponding Carreau-Yasuda dependences calculated according to Eq. 2 . index $n$ and the zero-shear rate viscosity were determined directly from the slope of each curve at high shear rates and their Newtonian plateau at low shear rates, respectively. These values were then used to calculate the two adjustable constants $a$ and $\lambda$ using Microsoft Solver ${ }^{\odot}$ in Excel $^{\odot}$ to obtain the best fit with the experimental data. The results are presented in Table 2. For pure MAPP it was not possible to apply Carreau-Yasuda approach and zero-shear rate viscosity cannot be precisely calculated.

The dependence of zero-shear rate viscosity on mixture composition for neat PP and 5, 10, and 50\% MAPP/PP is presented in Fig. 2. The range of approximate zero-shear rate viscosities for neat MAPP is shown by a bar. Experimentally determined zero-shear rate viscosity values are compared with additive viscosity $\eta_{0}$ add calculated according to the logarithmic mixing rule:

$$
\log \left(\eta_{0 \text { add }}\right)=M \log \left(\eta_{0 \text { MAPP }}\right)+(1-M) \log \left(\eta_{0 \text { PP }}\right)
$$

where $M$ is mass fraction of MAPP in the mixture and $\eta_{0}$ MAPP and $\eta_{0}$ PP are zero-shear rate viscosities of neat components. Because the zero-shear rate viscosity of MAPP cannot be well determined, two additive dependences are shown in Fig. 2 corresponding to $\eta_{0}$ MAPP upper and lower limit values. Theoretically, in the first approximation, the mixing rule is obeyed if there are no special interactions between mixture components. In our case it is clear that the MAPP/PP blends do not obey the mixing rule and zero-shear rate viscosity of MAPP/PP blends cannot be calculated using Eq. 3 for any mixture composition. The viscosity decrease with the addition of MAPP is strong, in particular for low MAPP concentrations at which the compatibilizer is actually present in composites: for example, the viscosity of the blend containing $5 \%$ MAPP is almost three times lower than pure PP viscosity, see inset of Fig. 2 and Table 2. In the following analysis of the rheological results obtained for composites, matrix viscosity will be taken equal to the corresponding MAPP/PP blend, as detailed in Table 2.

The viscosity of blends containing 2.5, 4.3, and 6.7 wt $\%$ MAPP (corresponding to 2, 3, and $4 \mathrm{wt} \%$ MAPP in composites) were not measured experimentally. For these blends, zero-shear rate viscosities (see inset of Fig. 2) and flow indexes were interpolated from the values measured

TABLE 2. Carreau-Yasuda parameters for MAPP/PP blends.

\begin{tabular}{llccc}
\hline Blend & $\eta_{0}(\mathrm{~Pa} \mathrm{~s})$ & $\lambda(\mathrm{s})$ & $n$ & $a$ \\
\hline PP & 3120 & 0.15 & 0.280 & 0.628 \\
$2.5 \% \mathrm{MAPP} / \mathrm{PP}$ & 2010 & 0.12 & 0.309 & 0.671 \\
$4.3 \% \mathrm{MAPP} / \mathrm{PP}$ & 1440 & 0.10 & 0.324 & 0.697 \\
$5 \% \mathrm{MAPP} / \mathrm{PP}$ & 1324 & 0.08 & 0.346 & 0.806 \\
$6.7 \% \mathrm{MAPP} / \mathrm{PP}$ & 925 & 0.07 & 0.344 & 0.737 \\
$10 \% \mathrm{MAPP} / \mathrm{PP}$ & 490 & 0.03 & 0.363 & 0.854 \\
$50 \% \mathrm{MAPP} / \mathrm{PP}$ & 102 & 0.03 & 0.64 & 0.777 \\
MAPP & $40-100$ & - & - & - \\
\hline
\end{tabular}




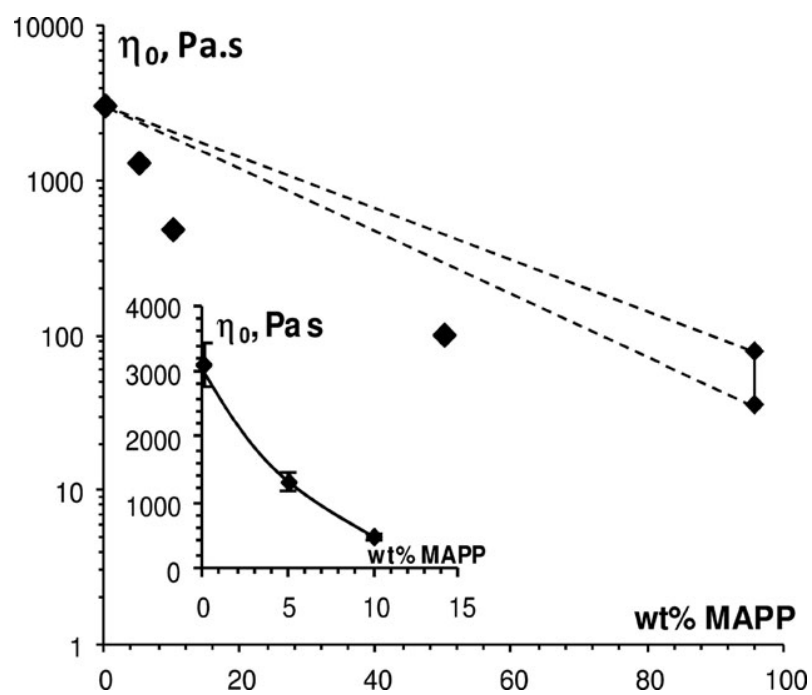

FIG. 2. MAPP/PP zero-shear rate viscosity calculated from CarreauYasuda model (Eq. 2) as a function of MAPP mass fraction, measured at $200^{\circ} \mathrm{C}$. The dashed lines correspond to the logarithmic mixing law (Eq. 3 ) considering highest and lowest zero-shear rate viscosity determined for the pure MAPP. Inset: zero-shear rate viscosity versus MAPP weight fraction at low MAPP concentrations. Line is given to guide the eye.

for 0,5 , and $10 \mathrm{wt} \%$ MAPP. Complete viscosity-frequency dependencies were built after applying time-temperature superposition, $\lambda$ and $a$ parameters were then calculated using Microsoft Solver ${ }^{\odot}$ in Excel $^{\odot}$. These values are presented in italic in Table 2 . These interpolated values will be used as matrix viscosity for the corresponding composites in the following.

\section{Rheological Behavior of Flax-Reinforced PP Composites: Cox-Merz Rule}

The viscosity of composites obtained from dynamic and capillary measurements as a function of frequency and shear rate, respectively, is presented in Fig. 3. Contrary to PP and PP/MAPP blends, the viscosity of composites is far from obeying Cox-Merz rule. Data obtained

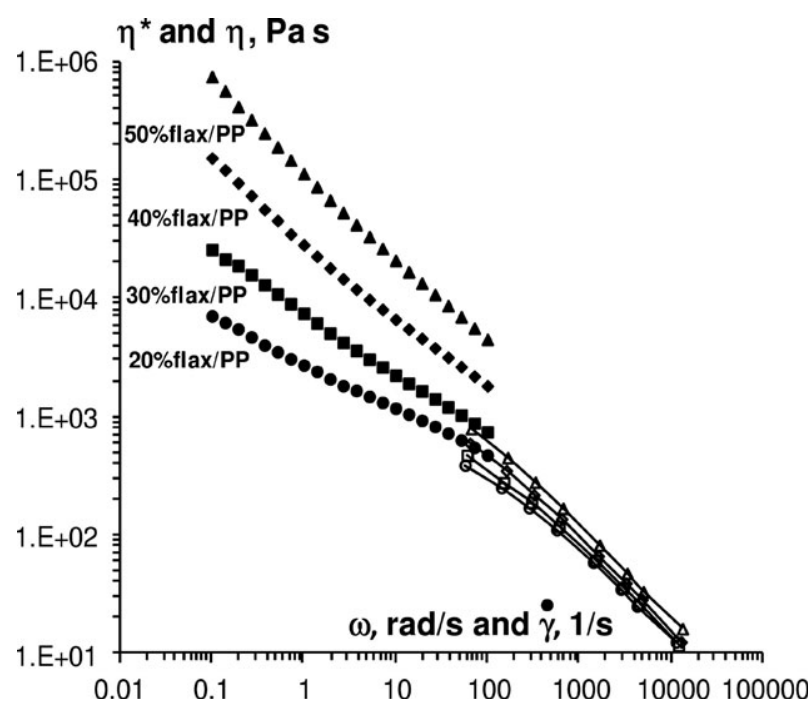

FIG. 3. Cox-Merz plot for the viscosity of flax/PP composites obtained from oscillation measurements (filled symbols), and capillary measurements (open symbols). Lines for capillary data are given to guide the eye.

with capillary rheometer are much lower than the ones obtained with oscillation measurements. A similar but less pronounced deviation of the Cox-Merz rule was observed by Mobuchon et al. [16] for $30 \mathrm{wt} \%$ glass fiber-reinforced PP. This behavior is explained by the very different composite structures formed during oscillation and shear flow in the capillary die: in plate-plate geometry, fibers are randomly oriented [23] (Fig. 4a), and in the capillary they are all aligned along the flow direction [24, 25] due to high mechanical stresses (Fig. 4b). Figure 4b shows that all fibers are oriented along the flow direction and are perpendicular to the cross-section. In addition, in the capillary a thin layer of polymer matrix is formed close to the wall acting as a "lubricant" and thus again decreasing viscosity $[16,26]$. In plate-plate geometry, composites behave as yield stress fluids at low frequencies due to fiber-fiber interactions and show shear thinning at higher
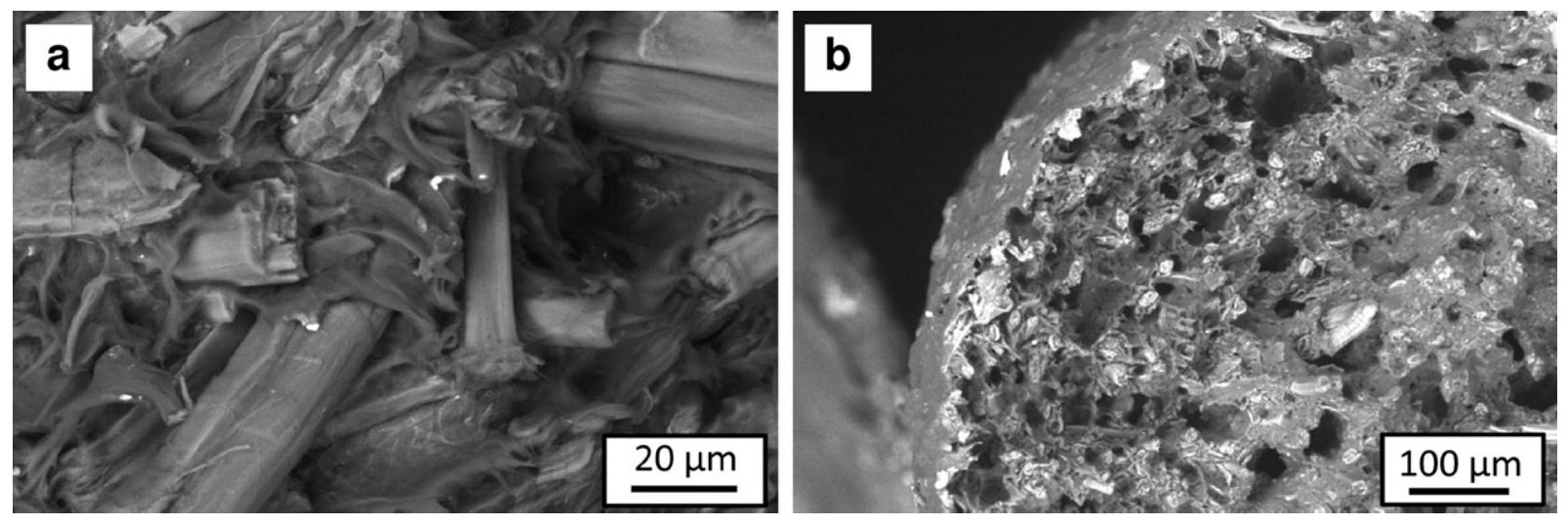

FIG. 4. Scanning electron microscopic images of cross-sections of $40 \mathrm{wt} \%$ flax/PP composite disc made for dynamic rheological experiments (a) and of $50 \mathrm{wt} \%$ flax/PP extrudate from capillary rheometer (b) where all fibers are oriented along the flow direction (perpendicular to the cross-section). 
frequencies; in capillary geometry, composites flow as strongly shear thinning fluids. In the following, oscillation and capillary results will be analyzed separately.

\section{Analysis of Dynamic Rheological Results}

The examples of elastic and viscous moduli $G^{\prime}$ and $G^{\prime \prime}$ of 30 and $50 \mathrm{wt} \%$ flax-based composites as a function of frequency are presented in Fig. 5 together with the moduli of pure PP. There is a remarkable difference in the viscoelastic response of composites when compared with neat PP (and also with MAPP/PP blends that are similar to PP but not shown not to overload the graph). PP shows a classical behavior of molten polymers with $G^{\prime \prime}>G^{\prime}$ almost over the entire frequency range studied and with power law exponents $G^{\prime} \sim \omega^{x}$ and $G^{\prime \prime} \sim \omega^{y}$ at low frequencies being: $x=1.3$ and $y=0.81$ (for $10 \% \mathrm{MAPP} / \mathrm{PP}$ $x=1.6$ and $y=1)$. The deviation from the theoretical exponents predicted by Maxwell approach describing viscoelastic response of a fluid, $x=2$ and $y=1$, is most probably related to polymer polydispersity. With the increase in fiber concentration beyond a particular volume fraction, composites behave like an elastic solid with $G^{\prime}>G^{\prime \prime}$. For $20 \mathrm{wt} \%$ flax/PP (not shown in Fig. 5), $G^{\prime \prime}$ is very slightly higher than $G^{\prime}$, and for $30 \mathrm{wt} \%$ flax/ PP $G^{\prime}=G^{\prime \prime}$ (Fig. 5).

The transition from fluid to solid-like behavior corresponds to the transition from semidilute to concentrated state which occurs when fiber volume fraction $f$ is roughly higher than $(L / D)^{-1}$. Let us estimate the "transition" volume fraction $f_{\text {semidilute/conc }}$ for flax-based composites. After processing, flax fibers are highly polydisperse [15] and thus both aspect ratios weighted in number and in surface should be considered. We know that for $20 \mathrm{wt} \%$ flax/PP the number and surface weighted aspect ratios are 5.2 and 9, respectively (see Table 1 ) [15]; $f_{\text {semidilute/conc }}$ is thus

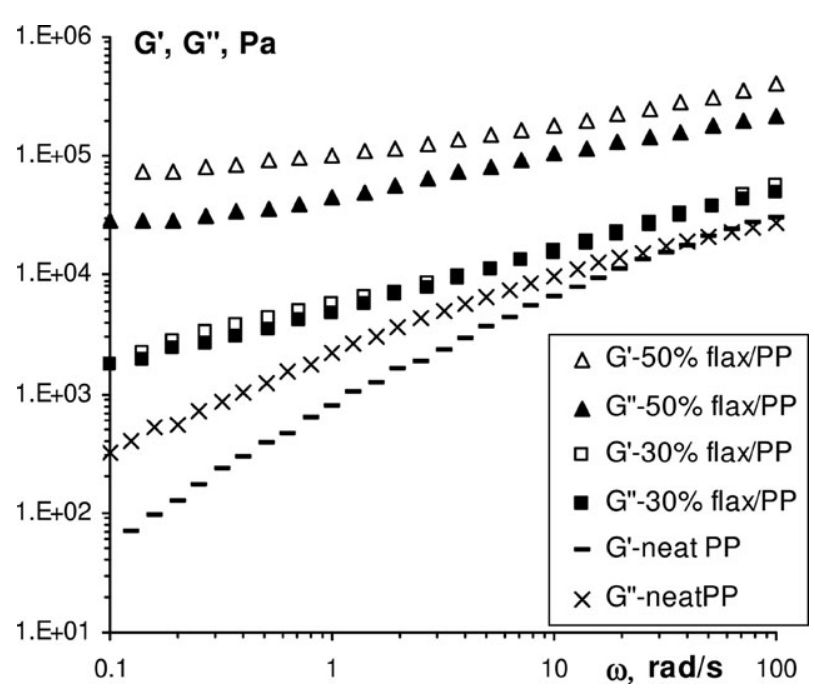

FIG. 5. Elastic and viscous moduli versus frequency for $30 \mathrm{wt} \%$ and $50 \mathrm{wt} \%$ flax/PP composites and neat polypropylene.
0.19 and 0.11 , respectively. The calculated volume fraction values of transition from semidilute to concentrated state are very close to fiber volume fractions corresponding to 20 and $30 \mathrm{wt} \%$ composites, 0.14 and 0.22 , respectively. This simple estimation confirms that indeed at fiber concentrations above $20 \mathrm{wt} \%$, flax fibers are forming a percolated "network" with a dominating elastic response.

The exponent $x$ in $G^{\prime} \sim \omega^{x}$ at low frequencies reflects the behavior of a system: $x=2$ indicates a liquid like and $x=0$, i.e., $G^{\prime}$ is frequency independent, indicates a solid like. The dependence of $x$ on fiber volume fraction in the composites studied is shown in Fig. 6; $40 \mathrm{wt} \%$ sisal/PP and 10\% MAPP/PP are also added for comparison. $x$ decreases with fiber volume fraction and becomes very low for $50 \mathrm{wt} \%$ flax/PP (fiber volume fraction $f=0.395$ ). It may be hypothesized that fibers in $50 \mathrm{wt} \%$ flax/PP composite are close to their maximal packing and suspension behavior becomes solid like. In the following, we shall demonstrate that indeed the volume fraction $f=0.4$ is close to the maximal packing of fibers with this aspect ratio. The exponent $x$ for $40 \mathrm{wt} \%$ sisal/PP is similar but higher than the one of $40 \mathrm{wt} \%$ flax/PP. The reason is that the aspect ratio of sisal fiber is lower than the one for flax (see Table 1). The transition from the semidilute to concentrated regimes for sisal-based composites is thus at higher volume fractions, giving a lower elastic response at the same fiber concentration.

As demonstrated in "Influence of MAPP on the rheological properties of PP/MAPP binary blends" section, MAPP strongly influences PP matrix viscosity which should be taken into account when analyzing dynamic viscosity experiments. To study the influence of fiber concentration on composite viscosity, it must be compared with the one of the corresponding MAPP/PP blend (see Table 1). Two examples of composite dynamic viscosity, 20 and 50 wt $\%$ flax/PP, are shown in Fig. 7. The viscosity

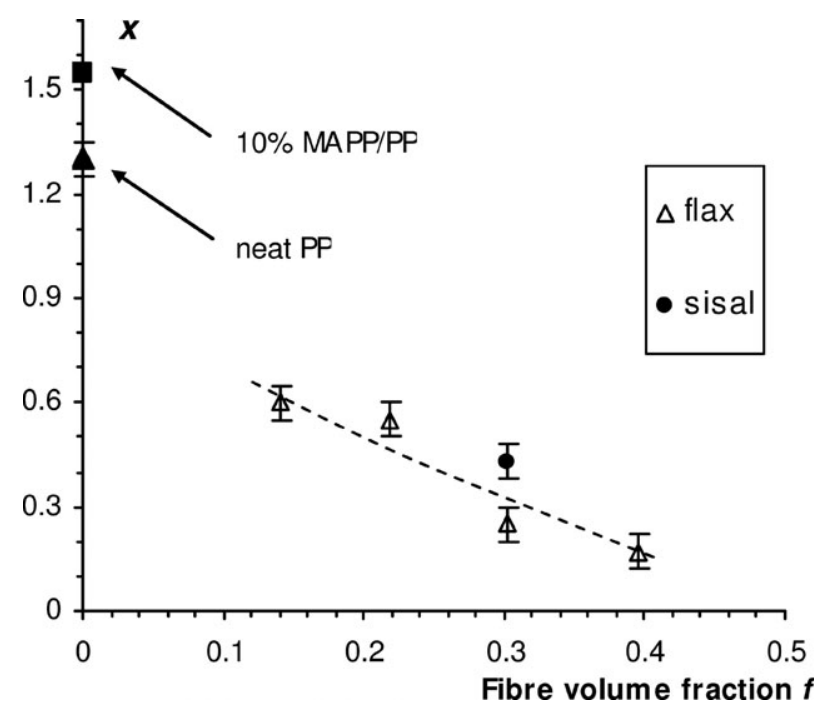

FIG. 6. Power law exponent $x$ in $G^{\prime} \sim \omega^{x}$ as a function of fiber volume fraction. Line is given to guide the eye. 
of $20 \mathrm{wt} \%$ flax/PP composite is overlapping with that of neat PP and the only difference is observed at low frequencies due to composite yield stress. Figure 7 shows that the presence of fibers does increase composite viscosity if comparing not with neat PP but with its corresponding MAPP/PP blend. Because for each fiber content different MAPP concentrations are used (see Table 1), the influence of fiber concentration on composite viscosity will be studied by defining a relative viscosity which is a ratio between the viscosity of a composite and its corresponding MAPP/PP blend. As far as composites and their corresponding matrices have different viscoelastic responses (composites are yield stress shear-thinning fluids and MAPP/PP blends demonstrate shear thinning at high frequencies with a Newtonian plateau at low ones), only zero-shear rate relative viscosity $\eta_{\text {rel }}$ will be used.

To calculate $\eta_{\text {rel }}$, zero-shear rate viscosity of each composite must be determined. MAPP/PP blends zero-shear rate viscosities are listed in Table 2. For composites, we used modified Carreau-Yasuda model that takes into account yield stress [27]:

$$
\eta *(\omega)=\frac{\sigma_{0}}{\omega}+\eta_{0}\left[1+(\lambda \omega)^{a}\right]^{(n-1) / a}
$$

where $\sigma_{0}$ is the yield stress, $\eta_{0}$ is the zero-shear viscosity, $\lambda$ is a characteristic time, $a$ is Yasuda parameter, and $n$ is the flow index reflecting shear-thinning. Equation 4 was successfully used for nanoclays-reinforced composites [28, 29] and more recently for hemp/PP composites [10]. Flow index $n$ was determined from the slope of each $\eta^{*}(\omega)$ curve at high frequencies and yield stress from stress-frequency dependence extrapolated to zero frequency. The model was thus reduced to three adjusta-

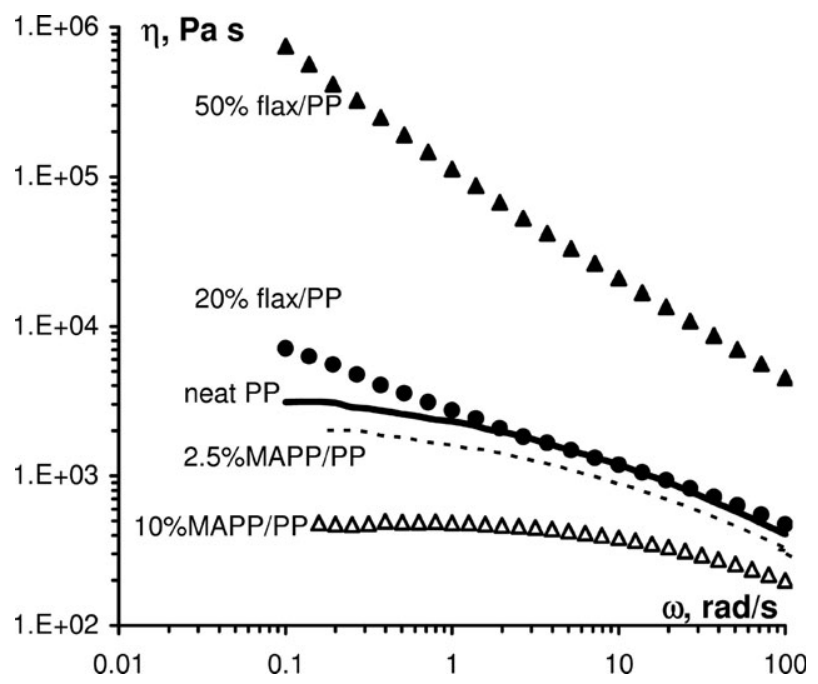

FIG. 7. Dynamic viscosity of 20 and $50 \mathrm{wt} \%$ flax/PP composites and their corresponding MAPP/PP matrices, $2.5 \% \mathrm{MAPP} / \mathrm{PP}$ (calculated) and 10\% MAPP/PP (experimental; see Table 2). Solid line corresponds to neat PP (experimental). ble parameters. Microsoft Solver ${ }^{\odot}$ in Excel $^{(}$was used to obtain the best fit with the experimental data (values listed in Table 3 and fits shown in Fig. 8).

Figure 8 shows that composite viscosity increases with increasing fiber concentration. The viscosity is greater for flax than for sisal-based composites because flax fibers have a higher aspect ratio (see Table 1). To quantitatively analyze the influence of fiber volume fraction on composite viscosity and to check the applicability of theories developed for suspensions in semidilute and concentrated regime, we used the Krieger-Dougherty model [30]:

$$
\eta_{\mathrm{rel}}=\left(1-f / f_{\mathrm{m}}\right)_{\mathrm{m}}^{-[\eta] f}
$$

where $\eta_{\text {rel }}$ is the relative viscosity as defined above, $f_{\mathrm{m}}$ is maximal packing volume fraction which depends on particle aspect ratio and size polydispersity, and $[\eta]$ is the intrinsic viscosity. Zero-shear rate viscosities of PP/MAPP blend and of the composites used for the calculation of relative viscosity are listed in Tables 2 and 3, respectively. The dependence of relative viscosity on fiber volume fraction is presented in Fig. 9 for flax and sisal based composites. The best fit for flax fibers (solid line in Fig. $9, R^{2}=$ 0.97 ) corresponds to $f_{\mathrm{m}}=0.54$ and $[\eta] f_{\mathrm{m}}=4.58$. The result for sisal-based composite is also shown; the relative viscosity is slightly lower when compared with flax composite because of sisal lower aspect ratio. $[\eta] f_{\mathrm{m}}=4.58$ is similar to the values reported for wood-polymer composites: [ $\eta] f_{\mathrm{m}}$ was found equal to 4 by Marcovich et al. [31] for wood flour/PP composites and to 4.2 by Dong et al. [32] for nontreated wood fiber/polyethylene composites.

Maximal packing volume fraction $f_{\mathrm{m}}$ is an important parameter characterizing the particle volume fraction at which viscosity becomes infinite. $f_{\mathrm{m}}$ depends on particle shape, aspect ratio, polydispersity and order in packing. The densest possible packing for organized monodisperse spherical particles is 0.74 (cubic and hexagonal close packed). The higher the particle aspect ratio, the lower is maximal packing volume fraction: for example, $f_{\mathrm{m}}$ decreases from 0.641 down to 0.343 for aspect ratios varying from 1 (randomly packed monodisperse spheres) to 9.17 (prolate particles), respectively [33]. Different $f_{\mathrm{m}}$ values are reported for wood-polymer composites: 0.6 for sawdust/PP composites with particles aspect ratios from 1.5 to 2 [6]; 0.6 for wood fiber/polyethylene composites with an aspect ratio of 10 [32] and 0.4 for wood flour/PP composites [31].

TABLE 3. Fit values (Eq. 4) obtained for complex viscosity curves of flax- and sisal-based composites.

\begin{tabular}{llccccc}
\hline Sample & $f$ & $\sigma_{0}(\mathrm{kPa})$ & $\eta_{0}(\mathrm{kPa} \mathrm{s})$ & $\lambda(\mathrm{s})$ & $n$ & $a$ \\
\hline $20 \mathrm{wt} \%$ flax/PP & 0.14 & 0.40 & 5.50 & 2.04 & 0.56 & 0.43 \\
$30 \mathrm{wt} \%$ flax/PP & 0.219 & 1.6 & 18.96 & 7.73 & 0.51 & 0.63 \\
$40 \mathrm{wt} \%$ flax/PP & 0.303 & 12.96 & 77.86 & 6.9 & 0.43 & 0.39 \\
$50 \mathrm{wt} \% \mathrm{flax} / \mathrm{PP}$ & 0.395 & 68.01 & 175.06 & 2.7 & 0.34 & 0.44 \\
$20 \mathrm{wt} \% \mathrm{sisal} / \mathrm{PP}$ & 0.14 & 0.39 & 5.5 & 10.16 & 0.62 & 0.45 \\
$40 \mathrm{wt} \%$ sisal/PP & 0.303 & 3.03 & 44.59 & 31.26 & 0.54 & 3.52 \\
\hline
\end{tabular}




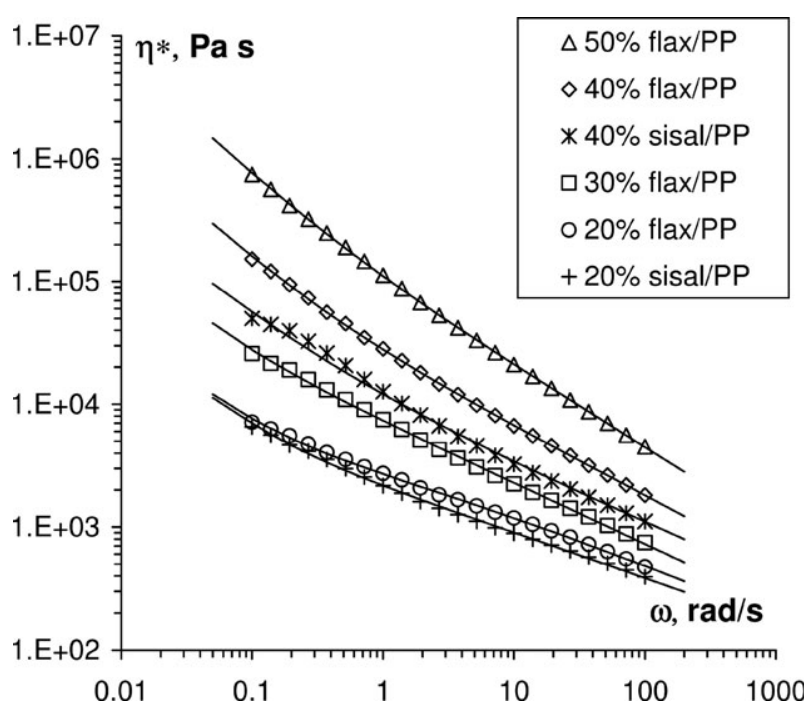

FIG. 8. Complex viscosity-frequency dependences for flax and sisalbased composites. Solid lines are the values calculated according to Eq. 4 with fit parameters as in Table 3. Experimental error is smaller or equal to symbol size.

It is interesting to check if various semi-empirical correlations developed for suspensions to estimate $f_{\mathrm{m}}$ as a function of aspect ratio can be used to predict $f_{\mathrm{m}}$ of our composites:

$$
\begin{gathered}
f_{\mathrm{m}}=0.54-0.0125(L / D)(\text { Kitano et al. [34]) } \\
f_{\mathrm{m}}=\frac{2}{0.321(L / D)+3.02} \text { (Mueller et al. [33]). }
\end{gathered}
$$

The calculation of the exact $f_{\mathrm{m}}$ value for the composites studied is rather delicate because of high fibers polydispersity and variation of aspect ratio as a function

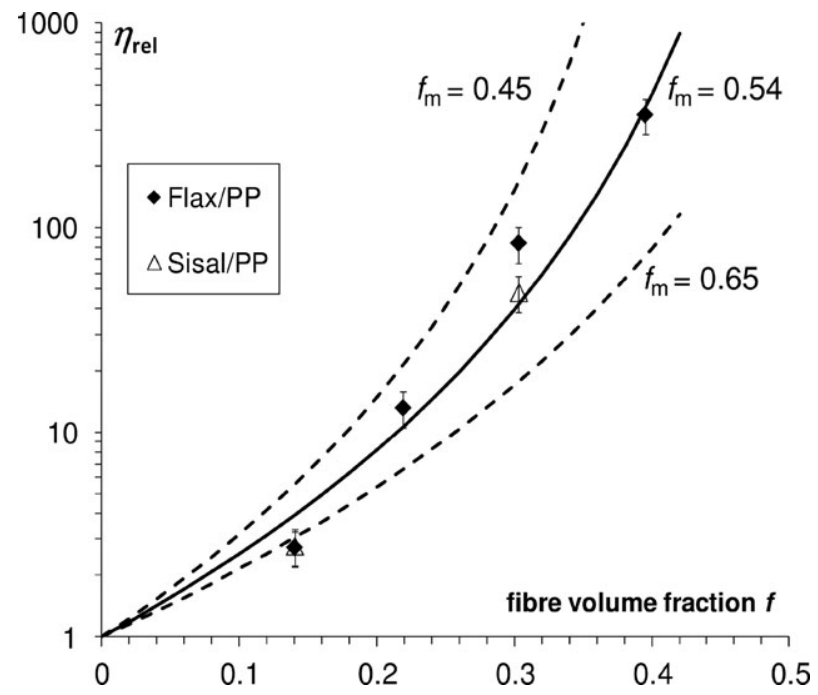

FIG. 9. Relative viscosity as a function of fiber volume fraction for flax/PP and sisal/PP composites. Solid line is the best fit to Eq. 5 for flax/PP composites with $f_{\mathrm{m}}=0.54$. The bars are for $20 \%$ error. Dashed lines correspond to $E q .5$ prediction with $f_{\mathrm{m}}=0.45$ and 0.65 . of fiber content (see Table 1). We calculated $f_{\mathrm{m}}$ values from Eqs. 6 and 7 using the maximal and minimal values of aspect ratios weighted in surface and in number, $(L / D)_{\mathrm{s}}$ $=9$ and $(L / D)_{\mathrm{n}}=5.2$, respectively (Table 1$)$ and compared with the $f_{\mathrm{m}}$ obtained from Krieger-Dougherty best fit. According to $E q .6, f_{\mathrm{m}}$ for flax fibers should vary from 0.42 to 0.48 and according to $E q .7$ from 0.33 to 0.43 . The $f_{\mathrm{m}}$ values resulting from $E q .7$ are too low, as fiber volume fraction in $50 \mathrm{wt} \%$ flax/PP composite is $f=$ 0.395 and this composite does not behave as a pure solid (see Figs. 5 and 6). The maximal volume fraction obtained for flax-based composites from fitting Eq. 5, $f_{\mathrm{m}}$ $=0.54$ (corresponding to $64.2 \mathrm{wt} \%$ ), is higher than the one estimated with $E q .6, f_{\mathrm{m}}=0.42-0.48$. The difference in $f_{\mathrm{m}}$ predicted by Kitano et al. [34] and Mueller et al. [33] and obtained as a best fit of Krieger-Dougherty model [30] can be explained by the high polydispersity of natural fibers size and shape [15] which increases the maximal packing volume fraction. Considering all approximations used, Eq. 5 appears to describe best $\left(R^{2}=0.97\right)$ the flax/PP composite viscosities. As predicted above in the analysis of the elastic modulus data (Figs. 5 and 6), fibers in $50 \mathrm{wt} \%$ flax/ PP composite $(f=0.395)$ are close to the maximal packing explaining its strong elastic response.

Table 3 shows that the higher is the fiber concentration in composites, the higher is the yield stress. The yield stress in natural fiber-reinforced composites had already been reported in literature for composites being in the concentrated regime, see for example, hemp/PP composites in Ref. [10]. As already mentioned above, composites studied here are in semidilute and concentrated regime. Surprisingly, several authors found that glass fiber-reinforced composites with a similar volume fraction $0.13(30 \mathrm{wt} \%)$ are not yield stress fluids even for fibers with a higher aspect ratio, around 20 [16] or around 14 [35]. Natural fiber specific properties such as an irregular surface, diameter variation along one fiber, fibrillation, capability to bend, etc. as well as high polydispersity in size and shape may be at the origin of strong fiber-fiber interactions responsible for the high yield stresses observed.

It was shown by Mueller et al. [33] and Heymann et al. [36] that for suspensions of solid particles it is possible to describe the yield stress versus particle volume fraction dependence in a way similar to Krieger-Dougherty model:

$$
\sigma_{0}=\sigma^{*}\left(\left(1-\frac{f}{f_{\mathrm{m}}}\right)^{-[\eta] f_{\mathrm{m}}}-1\right)
$$

where $\sigma^{*}$ is a fitting parameter related to the size of the particles $[33,36]$ and $f_{\mathrm{m}}$ and $[\eta] f_{\mathrm{m}}$ are the same as determined previously from fitting $E q$. 5. The dependence of the yield stress on fiber volume fraction for flax-based composites is shown in Fig. 10; the points for $20 \%$ and $40 \%$ sisal/PP are also added for comparison. The conclusions that can be made here are (i) yield stress is strongly 


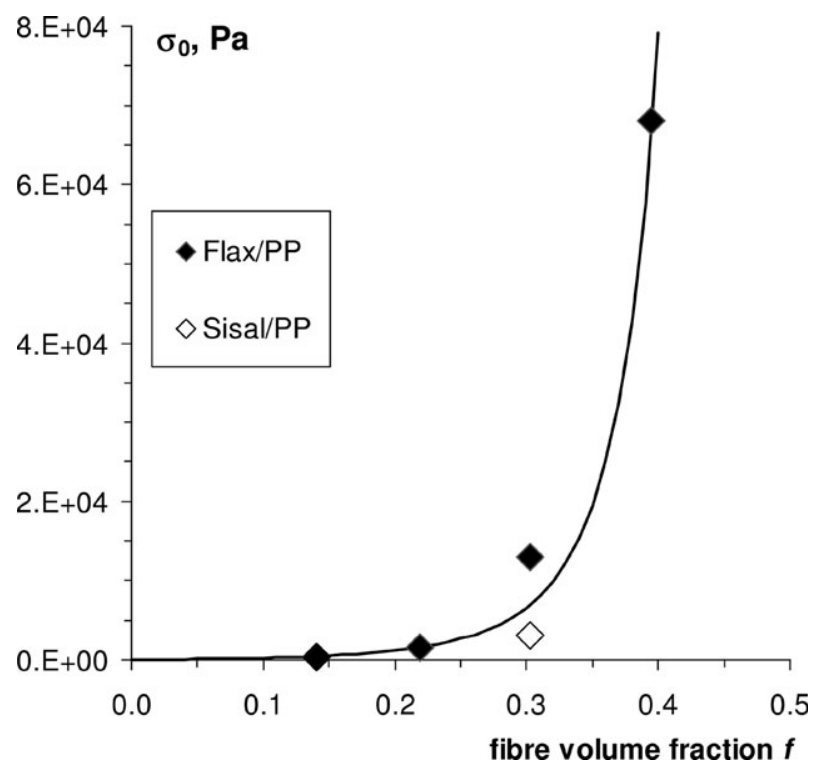

FIG. 10. Yield stress as a function of fiber volume fraction for flax/PP and sisal/PP composites. Solid line is the best fit to $E q .8$ for flax/PP composites with $f_{\mathrm{m}}=0.54,[\eta] f_{\mathrm{m}}=4.58$, and $\sigma^{*}=164 \mathrm{~Pa}$.

increasing with fiber volume fraction and can be approximated by Heymann et al. [36] approach using a modified Krieger-Dougherty model with $\sigma^{*}=164 \mathrm{~Pa}$ and (ii) the lower is fiber aspect ratio, the lower is the yield stress due to the less pronounced fiber interactions.

Flow index $n$ in Eq. 3 decreases with the increase in fiber volume fraction (Fig. 11). Here, only qualitative analysis can be performed because MAPP content in the matrix (MAPP/PP blend) differs from one flax concentration to another, and each matrix has a different shear thinning degree. Flow index increases with decreasing fiber aspect ratio (see flax vs. sisal in Fig. 11). Mueller et al.

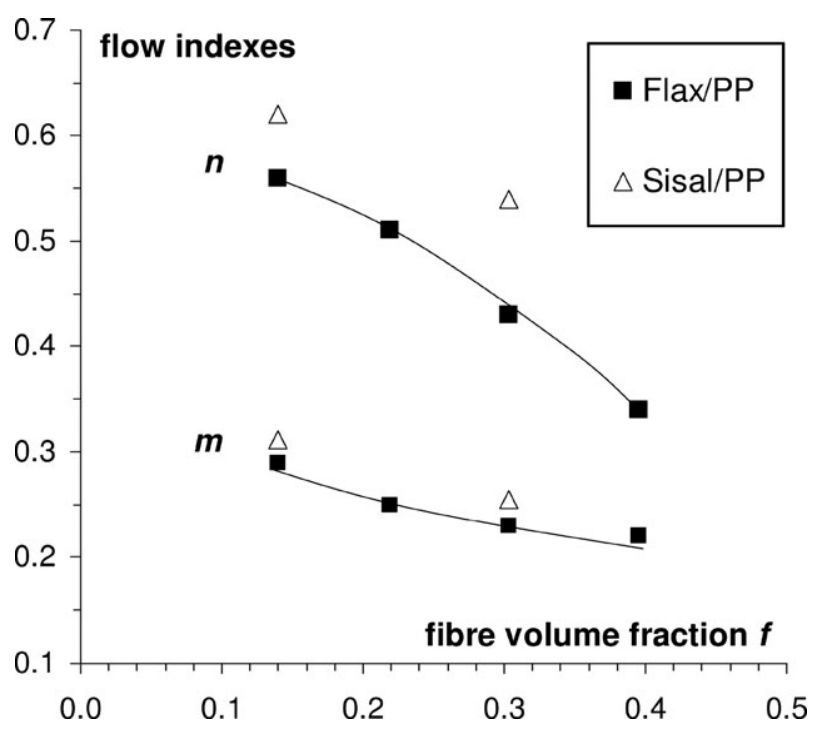

FIG. 11. Flow indexes $n$ (Eq. 4) and $m$ (Eq. 9) as a function of fiber volume fraction in flax/PP (filled symbols) and sisal/PP (open symbols) composites. Lines are given to guide the eye.
[33] suggest that the increased shear thinning in the presence of particles may result from local viscous heating between neighboring particles leading in a decrease in viscosity of the matrix, this effect being more pronounced for higher particle concentrations and aspect ratios.

\section{Analysis of Capillary Rheological Results}

Capillary rheology is a very useful tool to evaluate viscosity at high shear rates, comparable with the ones occurring during extrusion and injection molding. It is known and was already mentioned above that in the capillary die all fibers are oriented along the flow direction and that a layer of polymer is formed near the wall (see similar examples for glass fiber-reinforced composites in Refs. [24, 25, 35]. The macrostructure of molten composites in the capillary is thus very different when compared with plate-plate geometry (Figs. 3 and 4). Because of the fiber orientation, Cox-Merz rule is not obeyed (Fig. 3) and the rheological parameters determined with Eq. 4 (Table 3) cannot be used for viscosity analysis of capillary data. However, capillary viscosity data of MAPP/PP blends do obey Cox-Merz rule (Fig. 1) and thus the constants determined for this system with Eq. 2 (Table 2) can be used here to characterize matrix capillary viscosity.

The capillary flow of 20,40, and $50 \mathrm{wt} \%$ flax/PP composite is shown in Fig. 12; neat PP and $10 \mathrm{wt} \%$ MAPP/ $\mathrm{PP}$ blend corresponding to the matrix of $50 \mathrm{wt} \%$ flax/PP composite is also shown for comparison. Contrary to dynamic viscosity obtained with oscillation measurements, here the shapes of the curves of the composites and their matrices are very similar and practically do not depend on fiber content. The same trend had already been

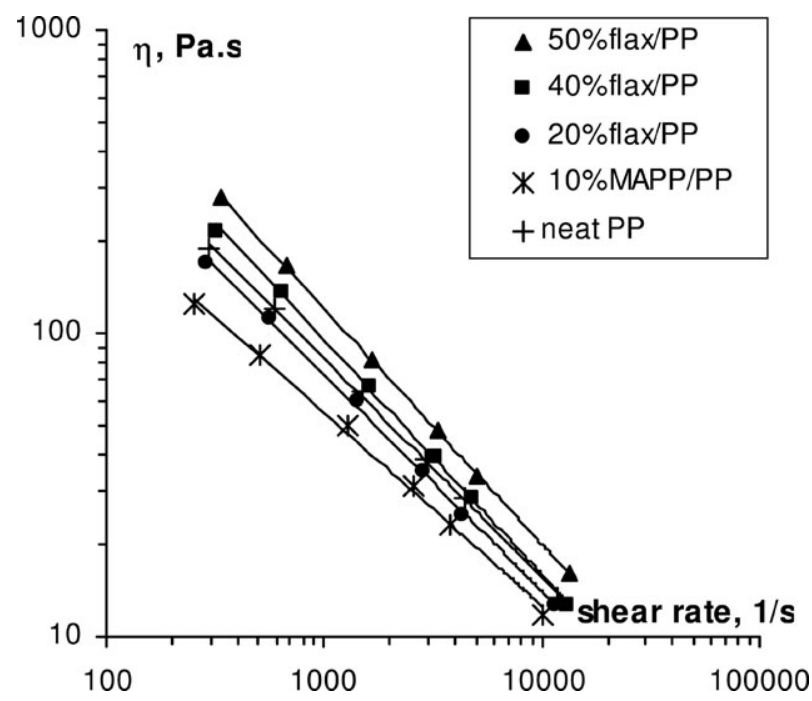

FIG. 12. Viscosity as a function of shear rate obtained with capillary rheometer for 20, 40, and $50 \mathrm{wt} \%$ flax/PP composites, neat PP and $10 \%$ MAPP/PP blend. Lines are power law approximations according to Eq. 9. 
reported for glass fiber-reinforced composites at high shear rates [35].

The viscosity of both MAPP/PP blends and of composites measured in a capillary rheometer can be described by the well known power law model:

$$
\eta(\dot{\gamma})=H \dot{\gamma}^{m-1}
$$

where the constant $H$ is consistency and $m$ is flow index, here at high shear rates. For MAPP/PP blends, $H$ is calculated as a best fit using flow indexes already determined for the whole viscosity curves in "Influence of MAPP on the rheological properties of PP/MAPP binary blends" section (in other words, for MAPP/PP blends $m=n$, see Table 2). The parameters determined for the composites and blends are listed in Table 4. $m$ and $H$ constants for MAPP/PP blends that were not experimentally obtained were calculated according to the following procedure. First, the dependences of $H$ and $m$ for the experimentally measured MAPP/PP blends (0, 5, 10, and $50 \mathrm{wt} \%)$ were plotted against MAPP concentration. Then the values for the matrices $2.5 \% \mathrm{MAPP} / \mathrm{PP}, 4.3 \% \mathrm{MAPP} / \mathrm{PP}$, and $6.7 \%$ MAPP/PP corresponding to 20, 30, and $40 \%$ flax based composites, respectively, were interpolated. They are indicated in italic in Table 4.

The dependence of flow index $m$ on fiber volume fraction in composites is shown in Fig. 11 together with results obtained in oscillation mode. Two facts can be noticed. First, the values of $m$ (capillary) are twice lower than the ones of $n$ (dynamic). Second, the decrease in $m$ with fiber content increase is much less pronounced than the decrease of $n$. The main reason for both observations is a very strong shear thinning in the capillary, when compared with oscillation experiments. Fiber orientation in the two geometries used is very different: whereas in the plate-plate geometry in oscillation mode fibers are completely disorganized at low stresses, fibers in the capillary die are all strongly oriented parallel to the die wall

TABLE 4. Fit parameters for composites and MAPP/PP blends according to $E q .9$.

\begin{tabular}{lcccr}
\hline & $\begin{array}{c}\text { \% MAPP in } \\
\text { MAPP/PP } \\
\text { Slend }\end{array}$ & $\begin{array}{c}\text { \% MAPP in } \\
\text { the composite }\end{array}$ & $m$ & \multicolumn{1}{c}{$H$} \\
\hline Neat PP & - & - & 0.28 & 11,803 \\
$20 \%$ flax & 2.5 & 2 & 0.29 & 10,071 \\
$30 \%$ flax & 4.3 & 3 & 0.25 & 14,542 \\
$40 \%$ flax & 6.7 & 4 & 0.23 & 19,854 \\
$50 \%$ flax & 10 & 5 & 0.22 & 25,828 \\
$20 \%$ sisal & 2.5 & 2 & 0.312 & 7754 \\
$40 \%$ sisal & 6.7 & 4 & 0.255 & 14,988 \\
$2.5 \%$ MAPP/PP & 2.5 & - & 0.309 & 8782 \\
$4.3 \%$ MAPP/PP & 4.3 & - & 0.324 & 7362 \\
$5 \%$ MAPP/PP & 5 & - & 0.346 & 6230 \\
$6.7 \%$ MAPP/PP & 6.7 & - & 0.344 & 5819 \\
$10 \%$ MAPP/PP & 10 & - & 0.363 & 4450 \\
$50 \%$ MAPP/PP & 50 & - & 0.640 & 350 \\
\hline
\end{tabular}

(compare Fig. 4a and b) due to high shear stresses. Shear thinning in the capillary is much less sensitive to the increase in fiber concentration because all fibers are aligned in the flow direction and fiber-fiber interactions are much less pronounced.

Relative consistency, $H_{\text {rel }}$, was used to analyze composite viscosity versus fiber volume fraction dependence using Krieger-Dougherty model [30] as previously described in "Analysis of dynamic rheological results" section for dynamic data:

$$
H_{\mathrm{rel}}=\left(1-f / f_{\mathrm{m}}\right)_{\mathrm{m}}^{-[\eta] f}
$$

where $H_{\text {rel }}=H_{\text {comp }} / H_{\text {matrix }}$ with $H_{\text {comp }}$ and $H_{\text {matrix }}$ being composite and the corresponding MAPP/PP blend consistency at high shear rates (Table 4). This approach was successfully used for the analysis of dynamic rheological properties of suspensions with particles of various shapes from 0.1 to 10 and volume fractions from 0 to 0.6 [33]. The results are shown in Fig. 13 together with the fit calculated according to Eq. 10. When compared with oscillatory data (Fig. 9), the increase in relative consistency (capillary data) with fiber volume fraction is much less steep than the increase in relative viscosity (dynamic data): when increasing $f$ from 0.14 to $0.4, \eta_{\text {rel }}$ increases 360 times whereas $H_{\text {rel }}$ only increases 4.5 times. The reason is that at low shear rates (plate-plate geometry) fibers are not aligned and the probability of their interactions is very high. This is clearly seen in Fig. 4a. As a consequence, dynamic viscosity and hence relative viscosity strongly increase with fiber concentration due to increasing fiber interactions. In the capillary die, fibers are strongly aligned and for the volume fractions used the distance between them should be higher or comparable

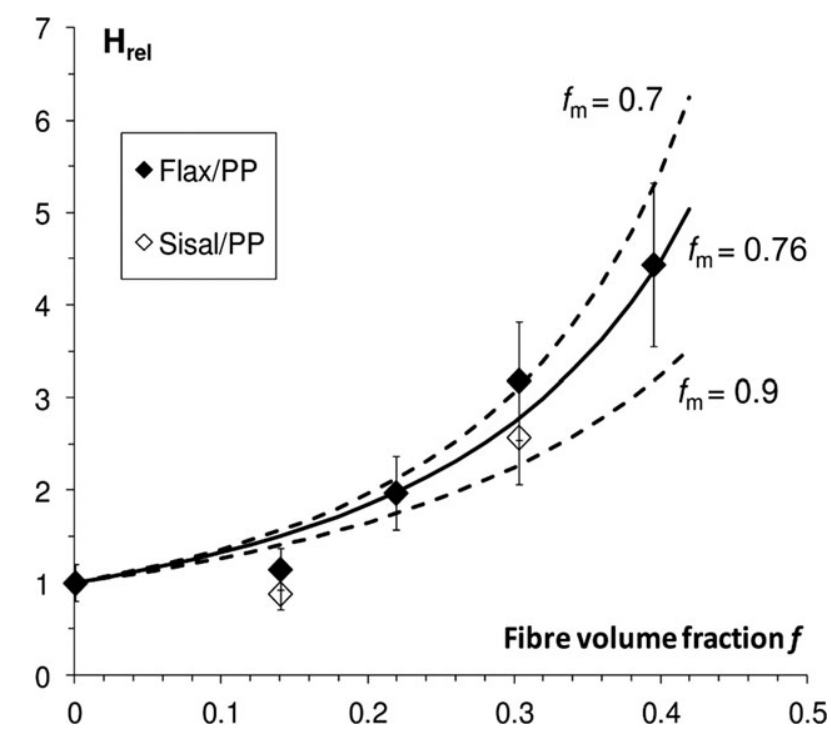

FIG. 13. Relative consistency as a function of fiber volume fraction for flax/PP and sisal/PP composites. Solid line is the best fit to Eq. 10 for flax/PP composites with $f_{\mathrm{m}}=0.76$. The bars are for $20 \%$ error. Dashed lines correspond to $E q$. 10 prediction with $f_{\mathrm{m}}=0.7$ and 0.9 . 
with their diameter (Fig. 4b). The probability of fiber collisions in aligned state is thus much lower than in disordered state. At the wall, where the shear rates are the highest, a layer of matrix is formed (Fig. 4b), thus further decreasing the resistance to shear and hence the relative consistency.

It is difficult to apply the Krieger-Dougherty approach [30] developed for randomly oriented particles in suspension to capillary data due to high alignment of both fibers and polymer matrix chains along the flow. It is obvious that the maximal packing volume fraction cannot be the same, the order of packing of the fibers being much higher in the capillary when compared with the plateplate geometry. The best fit $\left(R^{2}=0.951\right)$ was obtained with a maximal packing volume fraction $f_{\mathrm{m}}=0.76(82$ $\mathrm{wt} \%$ ) and an exponent $[\eta] f_{\mathrm{m}}=2$. In addition with the alignment of the fibers, this value of $f_{\mathrm{m}}$ is biased by the polymer layer formed near the wall which decreases the overall viscosity of the composites and thus artificially increases the maximal packing volume fraction. Other approaches including modeling of packing of fibers that are oriented and polydisperse in size and shape, and more experimental data are needed for performing an adequate analysis of the results obtained with capillary rheometry.

\section{CONCLUSIONS}

Dynamic and capillary rheological behavior of flaxand sisal-reinforced PP composites compatibilized with MAPP were studied for fiber concentrations ranging from 20 to $50 \mathrm{wt} \%$ and shear rates from $0.1 \mathrm{rad} \mathrm{s}^{-1}$ to 10,000 $\mathrm{s}^{-1}$. Both MAPP/PP blends and composites viscoelastic behaviors were studied in detail. It was demonstrated that the presence of MAPP in the PP matrix strongly decreases composites viscosity and counterbalances the increase in viscosity due to fibers. Although MAPP/PP blend viscosity obeys Cox-Merz rule, a large difference in dynamic and capillary viscosity of composites was recorded. The reason is random versus aligned fiber orientation in plateplate and die geometries, respectively.

All composites studied behave as yield stress shearthinning fluids. Dynamic viscosity of composites was modeled by a modified Carreau-Yasuda equation that takes into account the yield stress. The transition from semidilute to concentrated regime above $20 \mathrm{wt} \%$ for flax composite is accompanied by the formation of a "network" of fibers, which is reflected by the appearance of a yield stress; for $30 \mathrm{wt} \%$ composite the elastic modulus coincides with loss modulus over three frequency decades. Higher fiber concentrations lead to a dominant elastic behavior and higher yield stresses.

Classical approaches developed for suspensions of solid particles were applied for the analysis of zero-shear rate relative viscosity as a function of fiber volume fraction. The best fit with experimental data was obtained for the maximal packing fraction $f_{\mathrm{m}}=0.54$ using the Krieger-Dougherty model. The difference between $f_{\mathrm{m}}=$
0.54 obtained and $f_{\mathrm{m}}=0.42-0.48$ calculated with Kitano et al. approach was explained by the high polydispersity of flax fibers. Capillary results were also analyzed with the same approaches; it was demonstrated that strong fiber alignment in the flow direction and the formation of a polymer layer close to the wall lead to a significant increase in the maximal packing fraction, $f_{\mathrm{m}}=0.76$.

\section{ACKNOWLEDGMENTS}

NLM thanks the staff of Wageningen UR-Food and Biobased Research Institute for the technical support and scientific discussions. NLM is grateful to the European Polysaccharide Network of Excellence (EPNOE) for giving the opportunity to work at the Wageningen UR and to Carnot M.I.N.E.S. Institute for financing his post-doc. TB is grateful to the Industrial Chair in Bioplastics supported by Arkema, l'Oreal, Nestle, PSA, and Schneider Electric for stimulating research on natural fiber-reinforced composites.

\section{ABBREVIATIONS}

MAPP maleic anhydride-grafted polypropylene

PP polypropylene

WPC wood polymer composites

\section{REFERENCES}

1. A.K. Bledzki and J. Gassan, Prog. Polym. Sci., 24, 221 (1999).

2. A.K. Mohanty, M. Misra, and L.T. Drzal, Natural Fibres. Biopolymers and Biocomposites. Florida, USA: CRC Press, Taylor \& Francis Group, Boca Raton (2005).

3. J. Ganster and H.P. Fink, Cellulose, 15, 561 (2008).

4. T.Q. Li and M.P. Wolcott, Composites Part A, 35, 303 (2004).

5. T.Q. Li and M.P. Wolcott, Polym. Eng. Sci., 45, 549 (2005).

6. F. Godard, M. Vincent, J.F. Agassant, and B. Vergnes, J. Appl. Polym. Sci., 112, 2559 (2009).

7. S.N. Maiti, R. Subbarao, and M.N. Ibrahim, J. Appl. Polym. Sci., 91, 644 (2004).

8. K.J. Kim, S. Bumm, R.K. Gupta, and J.L. White, Compos. Interfaces, 15, 301 (2008).

9. D. Basu, A.N. Banerjee, and A. Misra, J. Appl. Polym. Sci., 46, 1999 (1992).

10. E. Twite-Kabamba, A. Mechraoui, and D. Rodrigue, Polym. Compos., 30, 1401 (2009).

11. M.J.A. Van den Oever and M.H.B. Snijder, J. Appl. Polym. Sci., 110, 1009 (2008).

12. R.T. Steller, J. Appl. Polym. Sci., 97, 1401 (2005).

13. S. Mohanty, S.K. Verma, and S.K. Nayak, J. Appl. Polym. Sci., 99, 1476 (2006).

14. A. Le Duc, B. Vergnes, and T. Budtova, Composites Part A, 42, 1727 (2011).

15. N. Le Moigne, M. van den Oever, and T. Budtova, Composites Part A, 42, 1542 (2011). 
16. C. Mobuchon, P.J. Carreau, M.C. Heuzey, M. Sepehr, and G. Ausias, Polym. Comp., 26, 247 (2005).

17. M.H.B. Snijder and H.L. Bos, Compos. Interfaces, 7, 69 (2000).

18. M.A. Bibbo, S.M. Dinh, and R.C. Armstrong, J. Rheol., 29, 905 (1985).

19. H.L. Bos, J. Müssig, and M.J.A. van den Oever, Composites Part A, 37, 1591 (2006).

20. A. Gonzalez-Monteil, J. Keskula, and D.R. Paul, J. Polym. Sci. Part B: Polym. Phys., 33, 1751 (1995).

21. L.I. Shucai, P.K. Järvelä, and P.A. Järvelä, J. Appl. Polym. Sci., 71, 1649 (1999).

22. L.I. Shucai, P.K. Järvelä, and P.A. Järvelä, Polym. Eng. Sci., 37, 18 (1997).

23. G. Ausias, J.F. Agassant, and M. Vincent, J. Rheol., 36, 525 (1992).

24. R.J. Crowson, M.J. Folkes, and P.F. Bright, Polym. Eng. Sci., 20, 925 (1980).

25. S.E. Barbosa and J.M. Kenny, Polym. Eng. Sci., 40, 11 (2000).
26. J.M. Charrier and J.M. Rieger, Fibre Sci. Technol., 7, 161 (1974).

27. F. Berzin, B. Vergnes, and L. Delamare, J. Appl. Polym. Sci., 80, 1243 (2001).

28. W. Lertwimolnun and B. Vergnes, Polymer, 46, 3462 (2005).

29. W. Lertwimolnun and B. Vergnes, Polym. Eng. Sci., 46, 314 (2006).

30. I.M. Krieger and T.J. Dougherty, Trans. Soc. Rheol., 3, 137 (1959).

31. N.E. Marcovich, M.M. Reboredo, J. Kenny, and M.I. Aranguren, Rheol. Acta, 43, 293 (2004).

32. S. Dong, S. Sapieha, and H.P. Schreiber, Polym. Eng. Sci., 32, 1734 (1992).

33. S. Mueller, E.W. Llewellin, and H.M. Mader, Proc. R. Soc. A, 466, 1201 (2010).

34. T. Kitano, T. Kataoka, and T. Shirota, Rheol. Acta, 20, 207 (1981).

35. H.M. Laun, Colloid Polym. Sci., 262, 257 (1984).

36. L. Heymann, S. Peukert, and N. Aksel, Rheol. Acta, 41, 307 (2002). 